\title{
Rational allocation of Australia's research dollars: does the distribution of NHMRC funding by National Health Priority Area reflect actual disease burden?
}

\author{
Rebecca J Mitchell, Rod J McClure, Jake Olivier and Wendy L Watson
}

$\mathrm{B}$ urden of disease (BoD) estimates are used as summary measures of population health and have been used to inform resource allocation and prioritisation of research needs. ${ }^{1}$ While a number of factors can influence the assessment and allocation of research funding, BoD measures have been used to identify areas in which research may help reduce the disease burden in the longer term. ${ }^{1,2}$

Rational allocation of expenditure for medical research implies a match between the distribution of funds, the BoD (as measured by the incidence or prevalence of a health condition and its social and economic costs), and evidence that new scientific discoveries, therapies or prevention strategies will improve outcomes or reduce the incidence of a condition. ${ }^{1,2}$

In Australia, since the late 1980s, a series of health policy documents has been developed to guide allocation of government resources. ${ }^{3}$ One of the most influential policy decisions has been to identify National Health Priority Areas (NHPAs). ${ }^{4}$ There are currently eight NHPAs: cardiovascular disease (CVD), cancer, injury, mental health, diabetes, asthma, arthritis and musculoskeletal conditions, and obesity. (Obesity has only recently been designated an NHPA and is not included in our analysis.)

The National Health and Medical Research Council (NHMRC) is the princi-
Abbreviations
BoD Burden of disease
CVD Cardiovascular disease
DALY Disability-adjusted life-year
NHMRC National Health and Medical Research Council
NHPA National Health Priority Area
SRDC Strategic Research Development Committee
YLD "Healthy" years of life lost due to disability
YLL Years of life lost due to premature mortality

\section{ABSTRACT}

Objectives: To explore National Health and Medical Research Council (NHMRC) funding for each National Health Priority Area (NHPA) over time and by grant type, and to quantify the relationship between grants awarded and a range of measures of societal burden of disease (BoD).

Design and setting: We conducted a retrospective analysis of NHMRC funding for each NHPA from 2000 to 2008 to assess the strength of correlation between level of NHMRC funding and contribution of each health condition to BoD. Information on mortality, incidence, prevalence, "healthy" years of life lost due to disability (YLD), years of life lost due to premature mortality (YLL) and disability-adjusted life-years (DALYs) was obtained from the 2003 Australian BoD study. Information on health system expenditure for each NHPA was obtained from an Australian Institute of Health and Welfare report.

Main outcome measures: Observed versus expected number of grants; amount of funding allocated to each NHPA; relative contribution of each NHPA health condition to BoD.

Results: 6099 new and continuing NHMRC grants were linked to NHPAs. Total NHMRC funding by NHPA was strongly correlated with YLL and DALYs, but there was no clear association between the amount of funding per NHPA and YLD or health system expenditure. Based on the proportional contribution of each NHPA health condition to total NHPA-related DALYs, a higher than expected number of grants was allocated to diabetes and cancer research, and a lower than expected number to injury and mental health research.

Conclusions: Some of Australia's NHPAs are better funded than others. The NHMRC could begin to redress this imbalance by allocating research and workforce development funding to less well developed research areas to ensure appropriate resourcing that is commensurate with their contribution to BoD.

MJA 2009; 191: 648-652

pal source of medical research funding in Australia. Key objectives of the NHMRC strategic plan ${ }^{5}$ are to fund the best and most relevant research, to support the best researchers, and to obtain evidence to support the application of research to practice. The NHMRC provides research funding in four main streams: training scholarships and fellowships; career awards; strategic awards and capacity-building; and research support.

Allocation of research funding has been analysed in several international studies. ${ }^{2,6,7}$ There has been only one previous Australian study, ${ }^{8}$ which compared the relationship between NHMRC funding and $\mathrm{BoD}$ measures based on data from the first Australian BoD study. ${ }^{9}$ The study found a significant relationship between NHMRC research funding and most BoD measures, but also showed that six NHPAs received only $29.5 \%$ of the disease-specific NHMRC funding, despite accounting for $70 \%$ of the total BoD in Australia. Allocation of research funding to NHPAs by grant type was not examined in the study, nor was the relationship between NHMRC funding and health system expenditure on NHPAs.

The aim of our study was to explore NHMRC funding for each NHPA over time and by grant type, and to quantify the relationship between grants awarded and a range of measures of societal BoD.

\section{METHODS}

We conducted a correlational study involving secondary analysis of Australian data sources. 


\section{Data sources}

NHMRC funding data for each NHPA over the period 2000-2008 (up to 28 August 2008) were obtained from the NHMRC's Research Management Information System. ${ }^{10}$ Information was available on awarded grants, including the grant title; grant type (NHMRC project grant, scholarship, training fellowship, Strategic Research Development Committee [SRDC] grant, NHMRC career award, NHMRC strategic award or career development award); the years that funding was provided; the total amount of funding received (excluding the goods and services tax); and the field of research (based on the Australian Bureau of Statistics Research Fields, Courses and Disciplines Classification ${ }^{11}$ ). Independent Research Institutes Infrastructure Support Scheme grants and equipment grants were excluded.

Information on the BoD was obtained from the 2003 Australian BoD study, ${ }^{12}$ which used methods initially developed for the global BoD study, ${ }^{13}$ with refinements developed in the original Victorian and Australian BoD studies.9,14,15 The measure used by these studies to quantify the impact of disease and injury at the population level was the disability-adjusted life-year (DALY). This measure extends the notion of potential years lost due to premature death to incorporate equivalent years lost due to poor health and disability. The DALY, therefore, allows the comparison of health losses due to conditions with varying distributions of mortality and morbidity. The disabilityadjusted years of life lost in a population for a given condition $x$ are calculated from the following formula:

$\operatorname{DALY}_{x}=\mathrm{YLL}_{x}+\mathrm{YLD}_{x}$

where $\mathrm{YLL}_{x}$ represents the years of life lost due to premature mortality attributable to health condition $x$, and $\mathrm{YLD}_{x}$ represents the "healthy" years of life lost due to disability attributable to health condition $x$.

Expenditure by the health system on each NHPA was obtained from an Australian Institute of Health and Welfare report, Health system expenditure on disease and injury in Australia, 2000-01. ${ }^{16}$ Expenditure estimates were based on the use of hospitals, aged-care homes, out-of-hospital medical services, pharmaceuticals, other professional services, dental services and research.

\section{Data analysis}

Data on the amount of NHMRC funding for each NHPA by grant type and year were extracted. The analysis was repeated for the proportion of total health system expenditure allocated to each NHPA. Poisson regression was used to examine any statistically significant changes over time in the proportion of NHMRC research funding provided to NHPAs overall and to each NHPA individually.

For the analysis of grant type by NHPA, the proportional contribution of each NHPA health condition to the total DALYs for all NHPAs was calculated and this was multiplied by the current number of grants awarded. This provided an estimate of the expected number of grants for each NHPA based on the contribution of each health condition to DALYs. Chi-squared tests were used to test for significant differences between observed and expected values.

Linear regression models were used to examine whether NHMRC research funding for each NHPA was proportional to BoD measures (ie, YLL, YLD, DALY) and health system expenditure. Ninety-five per cent confidence bands were generated for each regression line, with NHPAs outside the 95\% confidence bands indicating either significant under- or overfunding of the NHPA by the NHMRC. All analyses were performed using SAS software, version 9.1 (SAS Institute, Cary, NC, USA).

\section{RESULTS}

There were 25199 new and continuing NHMRC grants awarded in the period 2000-2008, representing a total allocation of about $\$ 3.3$ billion for medical research and workforce development. Of the total number of new and continuing grants, 6099
(24.2\%) were awarded for NHPAs, with a total value ranging from $\$ 94.6$ million in 2000 to $\$ 423.3$ million in 2008 . During the study period, neither the proportion of total NHMRC funding allocated to NHPAs nor the proportion of total NHPA funding provided to each individual NHPA (Box 1) changed significantly $(P=0.17$ and $P=0.41$, respectively).

For each grant type (except capacitybuilding grants), there were significant differences between the observed number of grants awarded and the number that would be expected if the proportional contribution of each NHPA health condition to DALYs was taken into account (Box 2). The NHPAs of injury and mental health received fewer scholarships, overseas training fellowships, career awards, research project grants and program grants than what would be expected based on their proportional contribution to total NHPA-related DALYs. The areas of CVD and cancer both received fewer SRDC grants than expected given their overall contribution to DALYs.

By contrast, diabetes was over-awarded in terms of scholarships, Australian training fellowships, career development awards, career awards, strategic awards and project grants. Cancer attracted more overseas training fellowships, career development awards, career awards, project grants and program grants awarded than what would have been expected. Asthma was overawarded in terms of Australian training fellowships, career awards and project grants. More career awards were given to CVD, more SRDC grants to mental health and more project grants to arthritis than what
1 Proportion of NHMRC research funding allocated for each National Health
Priority Area by year, 2000-2008

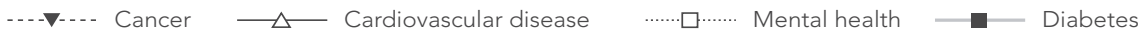

- . - Arthritic conditions and osteoporosis __ Injury _....... Asthma

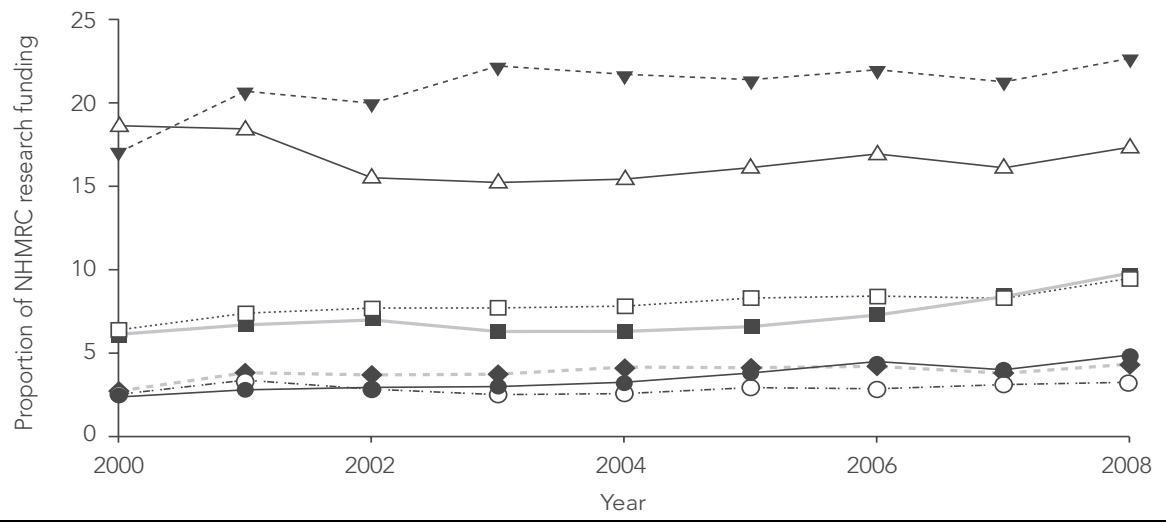

NHMRC $=$ National Health and Medical Research Council. 


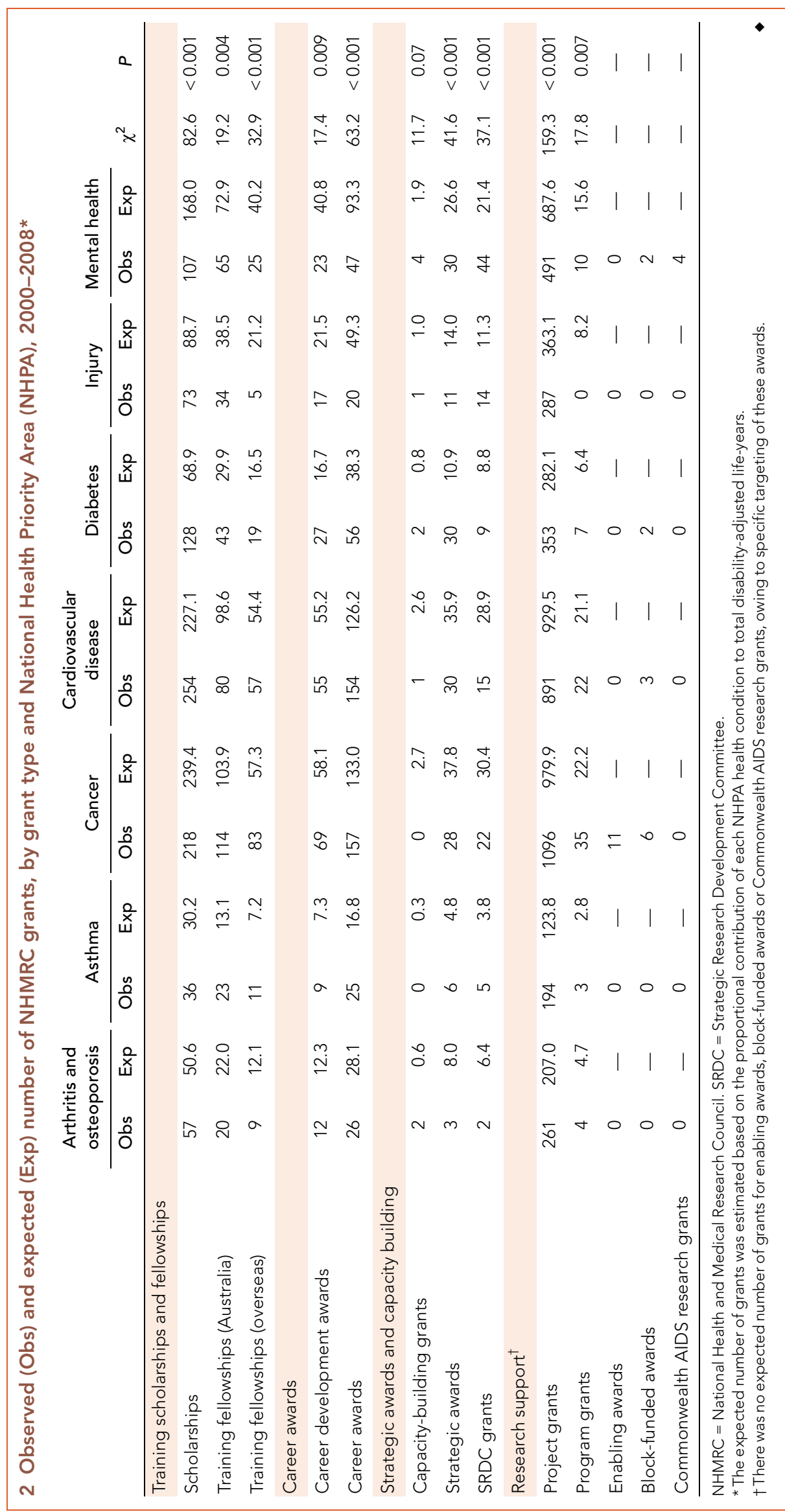

would have been expected based on their contribution to NHPA-related DALYs.

Regression models examined whether any measures of disease burden were associated with NHMRC research funding (Box 3, Box 4). The amount of NHMRC funding allocated to each NHPA was strongly correlated with YLL and DALYs, but not with YLD or health system expenditure. Diabetes and cancer each received more grants, and injury and mental health fewer grants, than would be expected if their respective proportional contribution to total NHPA-related DALYs were used as the determinant of NHMRC funding allocation.

\section{DISCUSSION}

The NHPA initiative was established with the intention of directing the allocation of national population health resources. ${ }^{4}$ The validity of the designation of the areas is supported by our finding that by 2003 Australia's NHPA health conditions accounted for $69.2 \%$ of the total national DALYs. In common with previous research, 2,6,8 we found that the relationship between funding for research and NHPAs varied depending on the BoD measure chosen for comparison. In our analysis, both DALYs and YLL were highly predictive of NHMRC funding allocation to NHPAs, indicating a significant relationship between NHMRC funding and these BoD measures, whereas health system expenditure and YLD were not predictive of NHMRC funding for NHPAs. There may be several reasons for this, including changes over time in the distribution and frequency of disabilities and in the treatment costs relating to some health conditions.

The NHPAs of injury and mental health appear to be underfunded based on YLL and DALY estimates, while cancer appears to be overfunded in terms of YLD and overall health system expenditure. Similar funding allocation discrepancies have been reported elsewhere - for example, World Health Organization funding for injury is substantially lower than would be expected given the burden of mortality and disability due to injury worldwide. ${ }^{7}$ Reasons for injury being underfunded may include a lack of perceived importance, competing priorities and limited advocacy efforts.

NHMRC grant funding for NHPAs increased overall between 2000 and 2008, but cancer and CVD research both received a larger proportion of funds than other NHPAs. While this primacy is to be expected, given that they are the leading 
3 Regression model of the relationship between NHMRC funding (2000-2008), YLL, YLD, DALY (2003), and health system expenditure (2000-01)
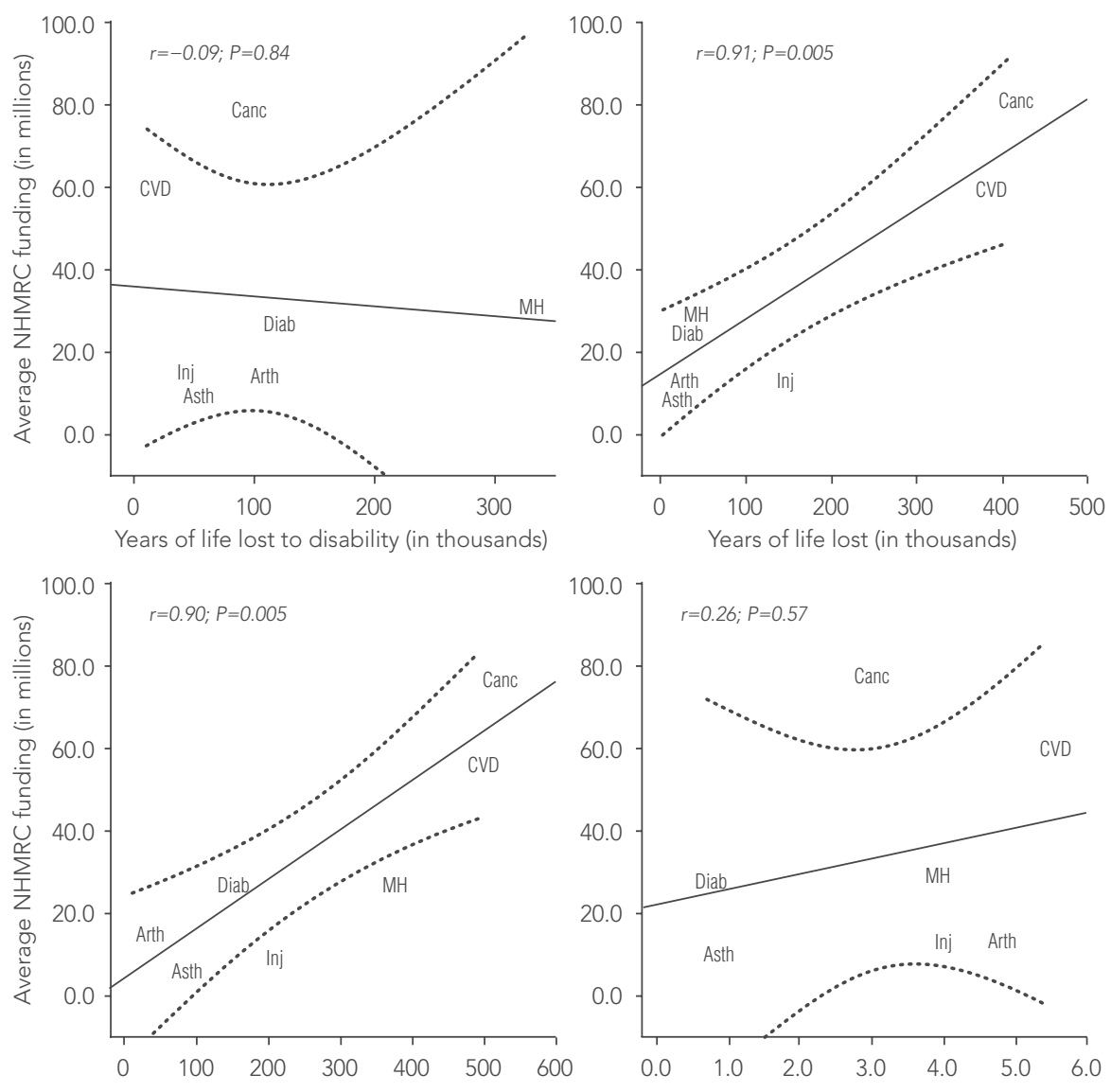

Disability-adjusted life-years (in thousands)

Arth $=$ arthritis. Asth $=$ asthma. Canc $=$ cancer. $C V D=$ cardiovascular disease. DALY = disability-adjusted life-year. Diab = diabetes. Inj = injury. $\mathrm{MH}=$ mental health. $\mathrm{NHMRC}=$ National Health and Medical Research Council. $\mathrm{YLD}=$ "healthy" years of life lost due to disability. YLL = years of life lost due to premature mortality.

causes of DALYs in Australia, our results suggest that cancer receives more funding than would be expected on the basis of its DALY contribution alone. When total funding is broken down by grant type, the overrepresentation of cancer is evident for research support and all levels of personnel support funding. A range of explanations may account for this. Cancer research may be more expensive to undertake for a given contribution to scientific knowledge. Being a more mature research area with more leading researchers, it may attract a higher proportion of funds than other NHPAs with less workforce capacity. It may also have a higher public and scientific profile, with greater potential to advocate for the importance of the field than other less resourced NHPAs.

The Wills review of the NHMRC ${ }^{17}$ clearly identified the NHMRC's responsibility to fund priority-driven research. However, the NHMRC's ability to rapidly respond to emergent research areas and changing policy directions is somewhat hampered by the mechanisms it uses to decide which research proposals to fund. While the NHPAs define priority areas for research, allocation of NHMRC funds remains largely determined by an investigator-driven, peer-reviewed, competitive process and is thus not well able to respond in a dynamic manner to new and emerging areas of research and policy focus. In an attempt to combat this limitation, the NHMRC has, from time to time, identified strategic and special initiatives for funding. For example, nine priority initiatives were identified for funding in 2009. ${ }^{18}$ Despite

4 Mortality, incidence, prevalence, health system expenditure and burden of disease (BoD) estimates for each National Health Priority Area

\begin{tabular}{|c|c|c|c|c|c|c|c|c|}
\hline & $\begin{array}{c}\text { Mortality } \\
2003\end{array}$ & $\begin{array}{c}\text { Incidence } \\
2003\end{array}$ & $\begin{array}{l}\text { Prevalence } \\
2003\end{array}$ & $\begin{array}{c}\text { Expenditure } \\
2000-01^{\star} \\
\left(\$ \text { million) (\%) }{ }^{\dagger}\right.\end{array}$ & $\begin{array}{l}\text { YLD } 2003 \\
\left({ }^{\prime} 000\right)^{\ddagger}(\%)^{\dagger}\end{array}$ & $\begin{array}{c}\text { YLL } 2003 \\
\left({ }^{\prime} 000\right)^{\ddagger}(\%)^{\dagger}\end{array}$ & $\begin{array}{l}\text { DALY } 2003 \\
\left({ }^{\prime} 000\right)^{\ddagger}(\%)^{\dagger}\end{array}$ & $\begin{array}{l}\text { BoD due to } \\
\text { premature } \\
\text { death }(\%)^{\S}\end{array}$ \\
\hline $\begin{array}{l}\text { Arthritis and } \\
\text { osteoporosis" }\end{array}$ & nd & nd & nd & 4634 (9.2\%) & $98.5(7.3 \%)$ & $7.0(0.5 \%)$ & 105.5 (4.0\%) & nd \\
\hline Asthma & 333 & 78493 & nd & 692 (1.4\%) & $59.1(4.4 \%)$ & $4.0(0.3 \%)$ & $63.1(2.4 \%)$ & nd \\
\hline Cancer** & 37222 & 470256 & nd & 2918 (5.8\%) & 87.5 (6.5\%) & 412.0 (32.2\%) & 499.4 (19.0\%) & $82 \%$ \\
\hline $\begin{array}{l}\text { Cardiovascular } \\
\text { disease }\end{array}$ & 48768 & 120847 & 770293 & 5479 (10.9\%) & 104.4 (7.7\%) & 369.4 (28.9\%) & $473.8(18.0 \%)$ & $78 \%$ \\
\hline Diabetes & 3590 & 97027 & 1170899 & 812 (1.6\%) & 111.5 (8.2\%) & $32.3(2.5 \%)$ & 143.8 (5.5\%) & $22 \%$ \\
\hline Injury & 10499 & 309023 & nd & 4013 (8.0\%) & 44.4 (3.3\%) & $140.6(11.0 \%)$ & 185.1 (7.0\%) & $76 \%$ \\
\hline Mental health & 1371 & 494618 & 3818170 & 3741 (7.5\%) & 327.4 (24.2\%) & $23.2(1.8 \%)$ & 350.5 (13.3\%) & $7 \%$ \\
\hline Total \% & & & & $44.4 \%$ & $61.6 \%$ & $77.2 \%$ & $69.2 \%$ & \\
\hline
\end{tabular}

DALY = disability-adjusted life-year. nd = no data. YLD = "healthy" years of life lost due to disability. YLL = years of life lost due to premature mortality.

* Total health system expenditure for the financial year 2000-01. Data source: Australian Institute of Health and Welfare. ${ }^{16}+$ Percentage of total.

$\ddagger$ Data source: Begg et al. ${ }^{12} \S$ Proportion of BoD due to premature death, with the remainder being due to years lived with disability.

१ Cost of arthritis and osteoporosis includes cost of all musculoskeletal conditions. ${ }^{\star \star}$ Cancer includes malignant and non-malignant neoplasms. 
these drawbacks, there are benefits to the competitive grant process. It ensures that the best research - that most likely to have important health outcomes - is funded, and that the research is conducted by motivated people with proven records of achievement.

The Wills review also recommended that the NHMRC be more responsive to national needs in terms of workforce capacity. ${ }^{17}$ Our analysis showed that some areas of research activity, in particular injury and mental health, receive relatively few grants for training scholarships, fellowships and career awards, while some areas, such as diabetes, receive relatively more scholarship grants and career awards. To combat this imbalance, the NHMRC could specifically allocate personnel support funds to under-resourced areas to build up workforce capacity. Targeted funding could support workforce development in emerging or underresourced areas so that researchers in these areas could compete with established areas that already have a large number of highprofile researchers attracting the bulk of NHMRC project and program grants. There are also other agencies, such as universities, whose recurrent scholarship or postdoctoral positions could assist with workforce capacity-building.

One limitation of our study was that the NHMRC is not the only source of research funding for NHPAs. Other sources include Australian Research Council (ARC) grants and funds from government agencies and philanthropic organisations. However, funding from these sources is relatively small, and excluding it from our analysis is unlikely to have affected the results.

Other limitations of our study relate to the possibility of error arising from secondary analyses of databases developed for administrative purposes. The accuracy of our results depended on the accuracy of the NHMRC's own databases. The accuracy of NHMRC coding by NHPA is unknown. NHMRC expenditure data are not adjusted for inflation, but this would not have affected comparisons between NHPAs. In comparing grants received by NHPAs and grant trends over time, we looked only at the number of grants awarded, not the amount of funding per grant. However, there is no evidence that a systematic difference in average cost of each grant between NHPAs would result in a bias that would compromise our conclusions.

\section{ACKNOWLEDGEMENTS}

Rebecca Mitchell was supported by an ARC-linkage postdoctoral fellowship (LP0990057). Jake Olivier was partially supported by the New South Wales Injury Risk Management Research Centre, with core funding provided by the NSW Department of Health, the NSW Roads and Traffic Authority and the Motor Accidents Authority. Wendy Watson was supported by the NSW Department of Health.

\section{COMPETING INTERESTS}

None identified.

\section{AUTHOR DETAILS}

Rebecca J Mitchell, MA(Psych), MOHS, PhD, Research Fellow ${ }^{1}$

Rod J McClure, PhD, FAFPHM, Director ${ }^{2}$

Jake Olivier, $\mathrm{PhD}$, Biostatistician 3,4

Wendy L Watson, BSc(Hons), MA, PhD, Senior

Research Fellow ${ }^{4}$

1 Department of Aviation, University of New South Wales, Sydney, NSW.

2 Monash University Accident Research Centre, Monash University, Melbourne, VIC.

3 School of Mathematics and Statistics, University of New South Wales, Sydney, NSW.

4 NSW Injury Risk Management Research

Centre, University of New South Wales,

Sydney, NSW.

Correspondence: r.mitchell@unsw.edu.au

\section{REFERENCES}

1 Michaud C, Murray C, Bloom B. Burden of disease - implications for future research. JAMA 2001; 285: 535-539.

2 Gross C, Anderson G, Powe N. The relationship between funding by the National Institutes Of Health and the burden of disease. $N$ Engl J Med 1999; 340: 1881-1887.

3 Mitchell R, McClure R. The development of national injury prevention policy in the Australian health sector and the unmet challenges of participation and implementation. Aust New Zealand Health Policy 2006; 3: 11.

4 Australian Institute of Health and Welfare. Health Priority Areas. http://www.aihw.gov.au/ nhpa/index.cfm (accessed Oct 2009).
5 National Health and Medical Research Council. National Health and Medical Research Council strategic plan 2007-2009. Canberra: NHMRC, 2007.

6 Lamarre-Cliche M, Castilloux A, LeLorier J. Association between the burden of disease and research funding by the Medical Research Council of Canada and the National Institutes of Health. A cross-sectional study. Clin Invest Med 2001; 24: 83-89.

7 Stuckler D, King L, Robinson H, McKee M. WHO's budgetary allocations and burden of disease: a comparative analysis. Lancet 2008; 372: 1563-1569.

8 Aoun S, Pennebaker D, Pascal R. To what extent is health and medical research funding associated with the burden of disease in Australia? Aust N Z J Public Health 2004; 28: 80-86.

9 Mathers C, Vos T, Stevenson C. The burden of disease and injury in Australia. Canberra: Australian Institute of Health and Welfare, 1999. (AlHW Cat. No. PHE 17.) http://www.aihw. gov.au/publications/phe/bdia/bdia.pdf (accessed Oct 2009).

10 National Health and Medical Research Council. NHMRC research funding datasets based on burden of disease and health issues. http:// www.nhmrc.gov.au/grants/dataset/issues/ index.htm (accessed Jan 2009).

11 Australian Bureau of Statistics. Australian Standard Research Classification (ASRC), 1998. Canberra: ABS, 1998. (ABS Cat. No. 1297.0.)

12 Begg S, Vos T, Barker B, et al. The burden of disease and injury in Australia 2003. Canberra: Australian Institute of Health and Welfare, 2007. (AlHW Cat. No. PHE 82.) http://www.aihw. gov.au/publications/index.cfm/title/10317 (accessed Oct 2009).

13 Murray C, Lopez A. The global burden of disease: a comprehensive assessment of the mortality and disability from diseases, injuries and risk factors in 1990 and projected to 2020. Cambridge, Mass: Harvard University Press, 1996

14 Vos T, Begg S. Victorian Burden of Disease Study: mortality. Melbourne: Victorian Government Department of Human Services, 1999.

15 Vos T, Begg S. Victorian Burden of Disease Study: morbidity. Melbourne: Victorian Government Department of Human Services, 1999.

16 Australian Institute of Health and Welfare. Health system expenditure on disease and injury in Australia, 2000-01. Canberra: AlHW, 2004. (AIHW Cat. No. HWE 26; Health and Welfare Expenditure Series No. 19.)

17 Wills PJ. The virtuous cycle. Working together for health and medical research. Canberra: Commonwealth of Australia, 1998.

18 National Health and Medical Research Council. 2009 project grant priority areas. http:// www.nhmrc.gov.au/grants/apply/projects/ index.htm\#pri (accessed May 2009).

(Received 4 Jun 2009, accepted 10 Aug 2009) 